\title{
Aniline incorporated silica nanobubbles
}

\author{
M J ROSEMARY, ${ }^{\mathrm{a}}$ V SURYANARAYANAN, ${ }^{\mathrm{a}}$ IAN MACLAREN ${ }^{\mathrm{b}+}$ and T PRADEEP ${ }^{\mathrm{a} *}$ \\ ${ }^{a}$ DST Unit on Nanoscience, Department of Chemistry and Sophisticated Analytical Instrument Facility, \\ Indian Institute of Technology Madras, Chennai 600036 \\ ${ }^{\mathrm{b}}$ Institute for Materials Science, Darmstadt University of Technology, Petersenstr. 23, 64287 Darmstadt, \\ Germany \\ ${ }^{+}$Present address: Department of Physics and Astronomy, University of Glasgow, Glasgow G12 8QQ, UK \\ e-mail: pradeep@iitm.ac.in
}

MS received 11 May 2006; revised 14 September 2006

\begin{abstract}
We report the synthesis of stearate functionalized nanobubbles of $\mathrm{SiO}_{2}$ with a few aniline molecules inside, represented as $\mathrm{C}_{6} \mathrm{H}_{5} \mathrm{NH}_{2} @ \mathrm{SiO}_{2} @$ stearate, exhibiting fluorescence with red-shifted emission. Stearic acid functionalization allows the materials to be handled just as free molecules, for dissolution, precipitation, storage etc. The methodology adopted involves adsorption of aniline on the surface of gold nanoparticles with subsequent growth of a silica shell through monolayers, followed by the selective removal of the metal core either using sodium cyanide or by a new reaction involving halocarbons. The material is stable and can be stored for extended periods without loss of fluorescence. Spectroscopic and voltammetric properties of the system were studied in order to understand the interaction of aniline with the shell as well as the monolayer, whilst transmission electron microscopy has been used to study the silica shell.
\end{abstract}

Keywords. Silica nanoshells; aniline; core-shell particles; spectroscopic studies; transmission electron microscopy; carbon onions.

\section{Introduction}

Incorporation of organic molecules such as dyes inside solid matrices is an attractive topic of research because of the photostability and fluorescence quantum yield ${ }^{1-3}$ of the modified materials. An approach in this regard is to incorporate molecules inside silica spheres, ${ }^{4,5}$ the advantage of this kind of nanoscopic containers is that they can be used to control the environment of the molecule. The molecule can be protected from unwanted chemical reactions and the cavity provides a rigid environment for the trapped molecules. Colloidal dispersions of silica shells are optically transparent, providing an opportunity to study the behaviour of the molecules incorporated without excessive light scattering problems. Imhof et $a l^{6}$ have studied the incorporation of fluorescein isothiocyanate inside silica spheres where the principal objective was to increase the photostability of the dye molecule. Results of this study seemed to suggest an inhomogeneous distribution of the molecules inside the shell. Studies have been performed

*For correspondence on the excited state reactions of the photochemically important molecule, ruthenium tris(bipyridyl) dye inside silica shells, where the excited Ru(II) showed significant enhancement of phosphorescence yield and lifetime. ${ }^{7}$ Moreover, the dye reacted with molecules such as methylviologen. Bosma et $a l^{8}$ have synthesized colloidal poly (methyl) methacrylate (PMMA) particles, where fluorescent dyes are incorporated into the polymer network. There are some other interesting studies as well on molecules such as pyrene adsorbed on silica gel, where the molecule showed 'excimer like' emission in addition to the monomer emission for different surface coverages. ${ }^{9}$ The synthetic approach followed in all these studies was to allow the dye molecules to react with a silane-coupling agent such as 3-(aminopropyl) triethoxysilane (APS) followed by the addition of another silane reagent like tetramethoxysilane (TMS), which undergoes hydrolysis/condensation incorporating the dye inside the silica shell. In a different approach, Makarova et $a l^{10}$ introduced a new method wherein fluorescein isothiocyanate was adsorbed on a nanoparticle surface after which silica was grown on it, thereby allowing the molecule to remain inside 
the shell. Recently, Ostafin et $a l^{11}$ have studied the encapsulation of cascade blue dye at high concentrations and have shown that the fluorescence intensity of the dye trapped inside the nano-sized silica bubbles was higher than that observed in the free solution under comparable conditions.

Since these molecules are isolated and confined within a shell, they may show different properties as compared to the free molecules. Hence spectroscopy of these materials offer a fascinating area of research. Many of these materials can be used as sensors and fluorescence intensity-based sensors have been also made. ${ }^{12-14}$ We have incorporated a simple molecule, namely aniline, inside the nanobubble and characterized the material in detail with various analytical tools. Free aniline is fluorescent and its fluorescent intensity decreases linearly with decreasing $\mathrm{pH}^{15}$ Steady state fluorescence of the respective material was also studied in detail and the spectrum investigated as a function of $\mathrm{pH}$. Fluorescence anisotropy measurements showed a high value compared to that of free aniline indicating less rotational freedom for the molecule inside the silica bubble. Infrared studies were also conducted to find out the type of interaction existing between aniline and silica. The external surface of the shell was modified with a monolayer cover of stearic acid so that this material can be dispersed in diverse media.

\section{Experimental}

\subsection{Materials}

Chloroauric acid, trisodium citrate, aniline and stearic acid were purchased from $\mathrm{CDH}$ fine chemicals, India. Aniline was used after distillation from zinc dust. (3-amino) propyl methyl diethoxysilane (APS) and tetra methoxysilane (TMS) were purchased from Aldrich and were used without additional purification. Ethanol and 2-propanol were purchased from $\mathrm{E}$ Merck. Carbon tetrachloride was purchased from Ranbaxy Chemicals, India. Ultra pure water was used for all the experiments.

\subsection{Synthesis of silica nanoshells}

Gold nanoparticles of size $15 \mathrm{~nm}$ were prepared using the Turkevich reduction method. ${ }^{16}$ In order to cover the gold particles with silica; a method adopted by Makarova et $a l^{10}$ was followed. To $200 \mathrm{ml}$ of the gold sol, $1 \mathrm{ml}$ of millimolar aqueous solution of ani- line was added under vigorous stirring and the solution was allowed to stand for $15 \mathrm{~min}$ so that complete complexation of aniline on gold surface took place. Next, $1.5 \mathrm{ml}, 1 \mathrm{mM}$ solution of freshly prepared APS was added to it with vigorous stirring. This mixture was again allowed to stand for around $15 \mathrm{~min}$ for complete complexation. A solution of active silica was prepared by adjusting the $\mathrm{pH}$ to $10-11$ of a 0.54 wt $\%$ of sodium silicate solution by progressive addition of a cation exchange resin, Dualite C 225-Na 14-52 mesh. $10 \mathrm{ml}$ of active silica thus prepared was added to $200 \mathrm{ml}$ of the surface-modified gold sol. The resulting mixture was allowed to stand for one day, so that the active silica polymerized on the surface of the gold particle to form $\mathrm{Au} @ \mathrm{SiO}_{2}$. Further growth of the silica shell was achieved by following the Stöbber ${ }^{17}$ method and the particle obtained by this method was of $90 \mathrm{~nm}$ size.

The solution thus obtained was centrifuged for around one hour and the particles were collected which were repeatedly washed with 2-propanol to make sure that no aniline was present on the surface of silica. This material was re-dispersed in a mixture of 2-propanol and water in the ratio, 4:1. To this, $2 \mathrm{M}$ sodium cyanide solution was added to remove the gold core and stirred for around 48 hours. The reaction between the gold core and sodium cyanide was monitored by UV/Vis spectroscopy. The dissolution of gold was confirmed by the disappearance of the gold plasmon peak. The formation of bubbles was confirmed from TEM images. By using carbon tetrachloride ${ }^{18}$ instead of sodium cyanide to remove the gold core, we observed carbon onion structures inside the silica shell in the TEM pictures. Later, when we extended this study for trapping other molecules such as ciprofloxacin, we could see the same kind of carbon onion structures inside the silica nanoshell (a detailed study of the microscopy of this onion material has been published separately). ${ }^{19}$ The material thus obtained was centrifuged at $2000 \mathrm{rpm}$ for around six hours, and the product collected was washed with 2-propanol and water and re-dispersed in water to yield aniline@ $\mathrm{SiO}_{2}$.

To disperse this material in organic solvents and also for easy storage and handling, we functionalized it by adding $1 \mathrm{mM}$ solution of stearic acid to the aqueous dispersion of aniline@ $\mathrm{SiO}_{2}$. We designate this monolayer protected material as aniline@ $\mathrm{SiO}_{2}$ @ stearate. This material can be freely dispersed, precipitated and stored. This is similar to monolayer protected $\mathrm{ZrO}_{2}$ core shell materials reported earlier. ${ }^{20}$ 


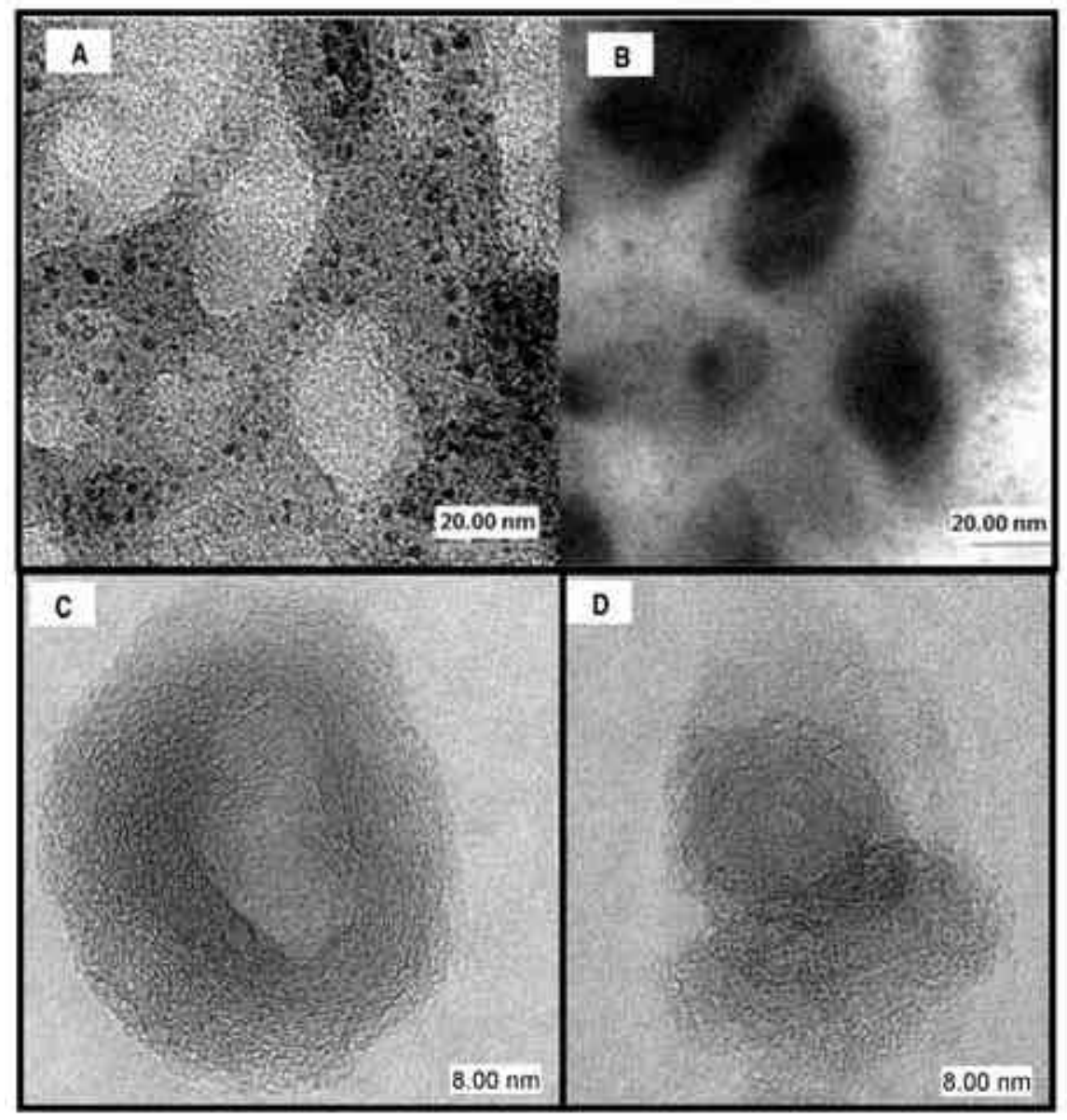

Figure 1. TEM images of bubbles. (A) Bright-field HRTEM image of a cluster of bubbles. (B) Energy filtered image of the same area using the $\mathrm{SiO}_{2}$ surface plasmon loss of $15 \mathrm{eV}$ showing the $\mathrm{SiO}_{2}$ shells very clearly. (C) Carbon onion structures inside the silica nanobubbles after $\mathrm{CCl}_{4}$ was used to remove the gold core for aniline@ $\mathrm{SiO}_{2}$. The graphitic spacing, corresponding to $0.3 \mathrm{~nm}$ is clearly visible. (D) The fusion of two carbon onion structures.

\subsection{Characterization}

UV/Vis absorption spectra were recorded using Perkin-Elmer Lambda 5 spectrometer and emission spectra were measured using F-4500 Hitachi spectroflourimeter. Anisotropy measurements were done using a Jobin-Vyon fluorolog instrument. FT-IR spectra were recorded with a Perkin-Elmer Spectrum One instrument using 5\% (by weight) $\mathrm{KBr}$ pellets. Transmission electron microscopic images were collected with a JEOL 3010 UHR TEM equipped with a Gatan Imaging Filter. Suspensions of the particles were dropped onto copper grid supported carbon-films and allowed to dry leaving particles dispersed on the carbon film. Cyclic voltammetry data were obtained from an electrochemical analyzer (CH Instruments Model 600A) in a standard three- electrode cell comprising a Pt disk (area $\left.=0.8 \mathrm{~mm}^{2}\right)$ as the working electrode, a platinum foil as the counter electrode and $\mathrm{Ag} / \mathrm{AgCl}$ as the reference electrode.

The mass spectrometric studies were conducted using a Voyager DE-PRO Biospectrometry Workstation (Applied Biosystems) MALDI-TOF MS instrument.

\section{Results and discussion}

As the oxide-protected nanoparticles have been adequately characterized, we present data only on the new materials. The hollow nanobubbles formed by the $\mathrm{CN}^{-}$removal method have an average shell diameter of around 10-20 nm (figure 1A and B) in agreement with the gold nanoparticles used in the 
synthesis. They are surrounded by roughly spherical amorphous silica shells, although it was difficult to determine the exact thickness since the shells were usually found in small clusters as here. Figure 1A shows a conventional bright field HRTEM image of the shells surrounded by the darker $\mathrm{SiO}_{2}$ shell material, the background comes from the $\mathrm{C}$ support film. Figure 1B shows an image of the same area recorded using the Gatan Imaging Filter to select electrons which have lost $15 \pm 2.5 \mathrm{eV}$. This has been shown in core-shell nanoparticles with $\mathrm{SiO}_{2}$ shells to highlight the $\mathrm{SiO}_{2}$ shell and appears to correspond to a surface plasmon of amorphous silica. ${ }^{21}$

Different studies of dye incorporation inside the silica shell by the same method have also reported nano shells of the same dimensions. ${ }^{10}$ As the material collected is white in colour and the solution is transparent in the visible region, we do not expect metal particles or adsorbed metal ions on the silica surface. No gold was detected in any of the analyses performed indicating that the ions were completely leached out from the particles.

We have analyzed the sample, aniline @ $\mathrm{SiO}_{2}$ made by the $\mathrm{CCl}_{4}$ removal method also. In this process nanoparticles of $\mathrm{Au}$ and $\mathrm{Ag}$ react with $\mathrm{CCl}_{4}$ forming $\mathrm{Au}^{3+}$ and $\mathrm{Ag}^{+}$, respectively. ${ }^{18,19}$ Amorphous carbon deposits in the process with the formation of $\mathrm{Cl}^{-}$in solution. The TEM Image of the formed material showed carbon onion-like structures inside silica shells (Figure 1C and D). The graphitic layers are clearly visible. The presence of carbon has been confirmed by electron energy loss spectroscopy and energy loss imaging. ${ }^{19}$ The formation of carbon onions is probably due to the deposition of amorphous carbon within the shells, which transforms to onions within the confinement of the nanocavity. The outer portion of the shell is amorphous in nature and inside that, in contact with the shell, the formation of the carbon onion structure starts.

These structures were not formed by electron beam irradiation on the amorphous carbon but were present at the initial stage of imaging itself. While silica parts are prone to electron beam induced damage, carbon structures are found to be stable. The formation of these structures gives more information on the shell structures. The shell appears plastic in nature and hence stretches and accommodates the carbon structures inside the shell.

Figure 2 gives the details of the microscopy of Au@silica as well as the nanoshell surrounding it. Image A shows the lattice resolved image of the gold silica core shell particle. The lattice of gold is clearly resolved. The gold core is clearly seen while the shell is faint. Images B, C and D show the fusion of isolated particles at different stages upon electron beam irradiation. It can be seen that as the two particles are nearby, they start fusing and forms a bigger particle upon electron beam irradiation. It confirms the fact that the silica shell deforms upon electron beam irradiation. This may be the reason for the formation of elongated structures as concluded from the TEM image of a single particle (figure 1C), while only spherical structures are expected to form by the leaching of gold from the core shell particles. The fusion of particles is very much clear from figure $2 \mathrm{~F}$ which was taken using $15 \mathrm{eV}$ loss electrons. Here, the shell can be clearly distinguished from the core. It is also important to note that the shell covers completely the resulting fused cores implying the plastic nature of the shell. Although fusion of nanoparticles is common under electron beam irradiation, it is shown here for core shell particles where the shell forms a continuous structure over the fused cores.

The absorption spectra of free aniline, Au@ aniline $@ \mathrm{SiO}_{2}$ and aniline $@ \mathrm{SiO}_{2}$ are shown in figure 3. Free aniline (trace a) has an absorption maximum around $280 \mathrm{~nm}$ due to $\pi \rightarrow \pi^{*}$ transitions. ${ }^{22}$ Spectrum (b) shows the absorption spectrum of Au@aniline@ $\mathrm{SiO}_{2}$. It shows a red shift in the surface plasmon resonance band from the typical value of $521 \mathrm{~nm}$ to $528 \mathrm{~nm}$ due to the modification of the gold surface, both due to aniline adsorption and due to the silica cover; the latter being more significant. ${ }^{23}$ The position and intensity of the gold surface plasmon depends on the particle size, and the optical and electronic properties of the system surrounding it. ${ }^{23}$ For $\mathrm{Au} @ \mathrm{SiO}_{2}$ core-shell structures, it is known that as the thickness of the silica cover increases; there is an increase in the absorption intensity and a red shift in the absorption maximum.

This red shift in plasmon absorption is attributed to the fact that aniline and APS are adsorbed on the gold surface. The encapsulated aniline molecules inside the silica shell after removing the gold core shows distinct aniline absorption at $280 \mathrm{~nm}$ (trace c). This shows that the shell acts as a stable container for this molecule. The supernatant, after precipitation of aniline@ $\mathrm{SiO}_{2}$ by centrifugation, did not show aniline features in the absorption spectrum.

Infrared spectroscopy was used to characterize the material as well as to look at the type of interactions possible for aniline inside the nanobubble. In the 


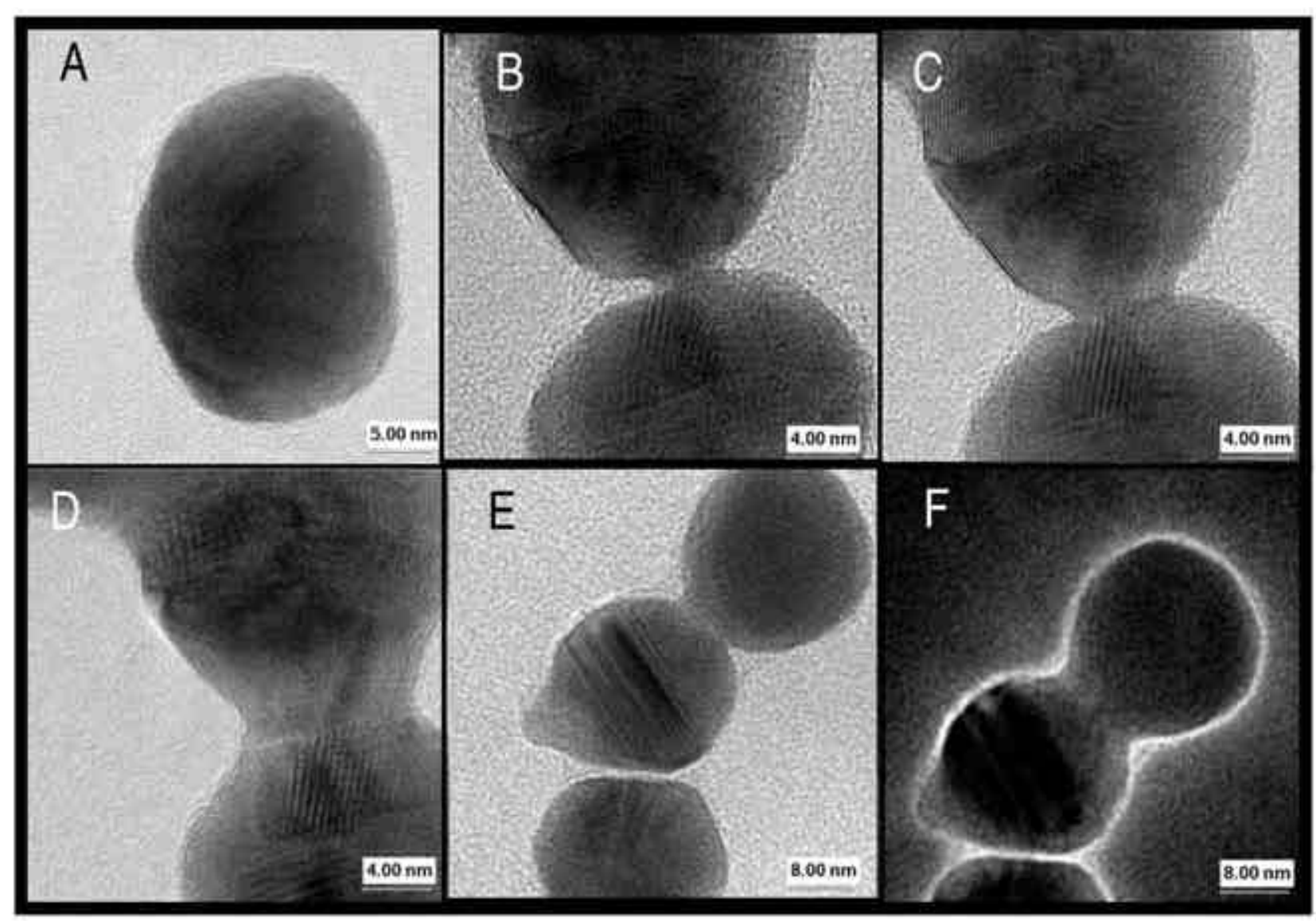

Figure 2. TEM images showing the fusion of $\mathrm{Au} @ \mathrm{SiO}_{2}$. (A) A lattice-resolved image of $\mathrm{Au} @ \mathrm{SiO} 2 .(\mathbf{B}),(\mathbf{C})$ and $(\mathbf{D})$ show particle fusion upon continuous electron beam irradiation; the images have been taken within 2 minutes of beam exposure. (E) An image of a collection of particles. (F) Image taken with $15 \mathrm{eV}$ loss electrons showing the shell structure.

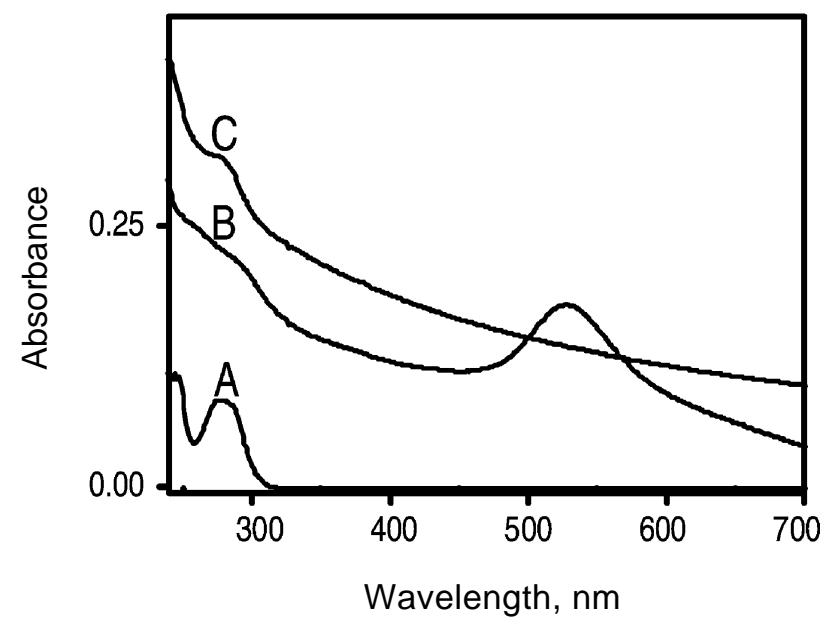

Figure 3. Absorption spectra of (A) free aniline, (B) $\mathrm{Au} @$ aniline@ $\mathrm{SiO}_{2}$ and $(\mathrm{C})$ aniline@ $\mathrm{SiO}_{2}$ in aqueous medium. The background present in (B) and (C) can be attributed to the formation of the thick shell and also due to the difference in refractive index between the medium and the silica shell. case of free aniline, the characteristic $\mathrm{N}-\mathrm{H}$ symmetric and asymmetric bands occur at $3370 \mathrm{~cm}^{-1}$ and $3458 \mathrm{~cm}^{-1}$, respectively (figure 4$){ }^{24}$ In the case of aniline@ $\mathrm{SiO}_{2}$ there is only a broad band at around $3442 \mathrm{~cm}^{-1}$, showing the presence of hydrogen-bonded water within the silica nanoshell. The bending mode of water and the $-\mathrm{NH}_{2}$ scissoring band are seen overlapped at $1617 \mathrm{~cm}^{-1}$ and an $\mathrm{NH}_{2}$ wagging band is seen at $657 \mathrm{~cm}^{-1}$ (figure 4). ${ }^{24}$ Apart from these a C-N stretching band at $1262 \mathrm{~cm}^{-1}$ as well as $\mathrm{Si}-\mathrm{O}-\mathrm{Si}$ asymmetric stretching band at $1083 \mathrm{~cm}^{-1}$ are also seen. ${ }^{24}$ Other than aniline and silica features, the spectrum also shows a sharp feature of amorphous carbon at $1384 \mathrm{~cm}^{-1}$ formed by the reaction of $\mathrm{CCl}_{4}$ and gold nanoparticles. ${ }^{18}$

We have also studied the fluorescence spectrum of aniline@ $\mathrm{SiO}_{2}$ (figure 5). Free aniline has an emission maximum around $340 \mathrm{~nm}^{25}$ whereas aniline@ $\mathrm{SiO}_{2}$ has a broad emission peak around $400 \mathrm{~nm}$. This behaviour can be understood by looking at the inter- 

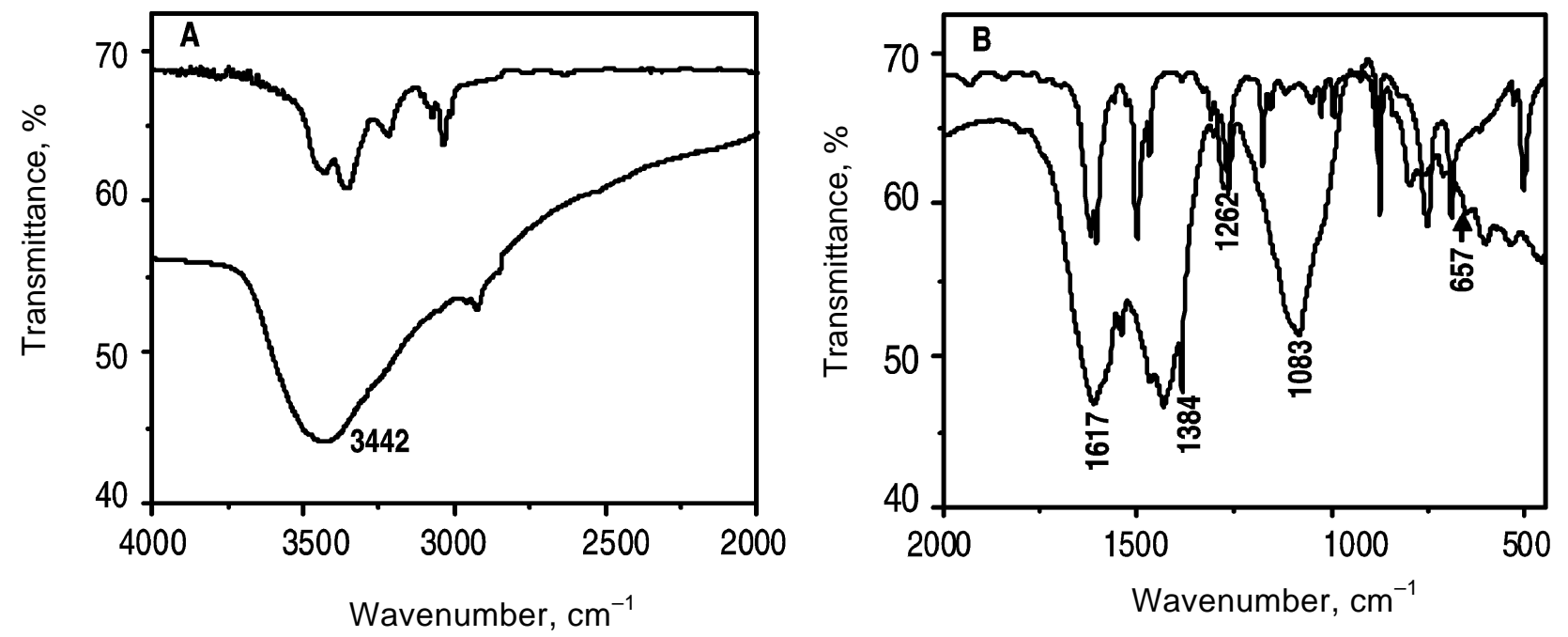

Figure 4. FT-IR spectrum of free aniline (upper trace) and aniline@ $\mathrm{SiO}_{2}$ (lower trace) in the high (A) and low (B) frequency regions. The aniline $@ \mathrm{SiO}_{2}$ sample was dried at $60^{\circ} \mathrm{C}$.

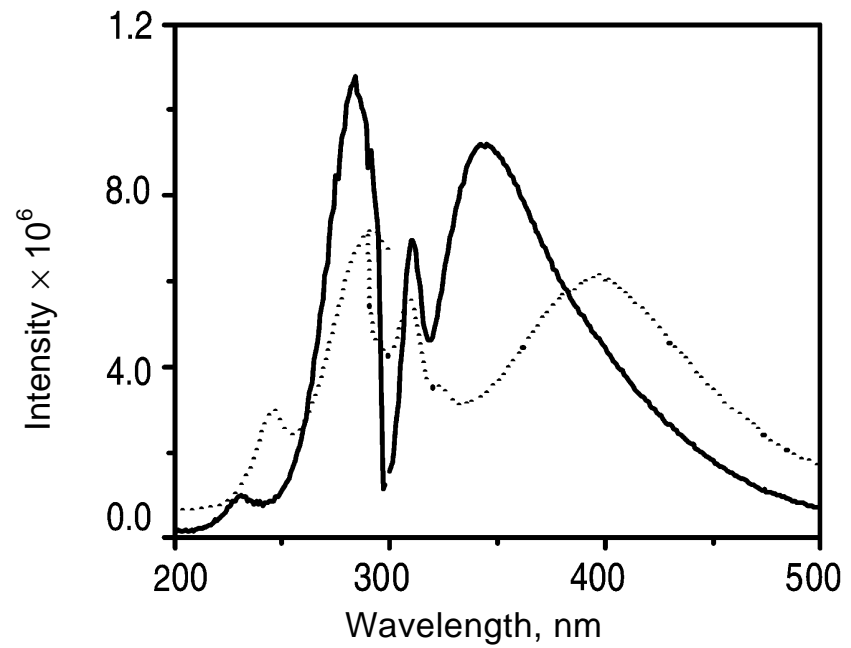

Figure 5. Excitation and emission spectrum of aniline@ $\mathrm{SiO}_{2}$ (dotted line) and free aniline in aqueous medium (solid line).

actions of the fluorophore with the solvent. There are two types of solvent effects; one is the general solvent effect which takes into account of polarizability as well as the dielectric constant of the solvent and can be approximately explained by the Lippert equation; and the specific solvent effect which arises due to the specific interactions like hydrogen bonding or charge transfer interactions. ${ }^{26}$ The red shift observed in the case of molecules incorporated inside the silica nanoshell shows a maximum value of 10 to
$20 \mathrm{~nm}$. For example, Ostafin et $a l^{11}$ has shown that cascade blue dye inside the silica nanobubble shows a decrease in intensity as well as a red shift of $10 \mathrm{~nm}$ in the emission spectrum compared to the free solution. ${ }^{11}$ A similar behaviour is found in the case of different concentrations of rhodamine $6 \mathrm{G}$ molecules loaded in silica. ${ }^{27}$ This kind of large red shifts are seen when specific interactions happen between the fluorophore and the solvent. ${ }^{26}$ Since there is a signature of hydrogen bonded water in the infrared spectrum, we suggest that aniline is hydrogen bonded to water within the nanoshell. Moreover hydrogen bond is reported for aniline when incorporated inside the silica matrices. ${ }^{28}$ Specific solvent-fluorophore interactions can happen in the ground state as well as in the excited state of a molecule. The absorption spectrum for aniline@ $\mathrm{SiO}_{2}$ shows a broadening in its peak position as well as a red shift in the excitation spectrum, suggesting that aniline is hydrogen bonded in the ground state itself.

Further studies showed that the fluorescence anisotropy of aniline@ $\mathrm{SiO}_{2}$ is around 0.1172 whereas for free aniline it is 0.0560 in aqueous solution at room temperature. This indicates that aniline@ $\mathrm{SiO}_{2}$ has a lesser rotational freedom as compared to free aniline. Anisotropy studies have been used for finding out the rotational freedom of different fluorophores in organized media. ${ }^{29,30}$ Tleugabulova $e t ~ a l^{31}$ have studied the binding of rhodamine $6 \mathrm{G}$ to silica spheres by using anisotropy decay (the system has a very high limiting anisotropy of $0 \cdot 38$ ). Also 3 -hydr- 
oxyflavone (3HF) in water at $35^{\circ} \mathrm{C}$ shows an anisotropy of 0.11 inside liposomes when compared to 0.06 in the aqueous solution. ${ }^{32}$

A solution of silica nanobubbles prepared using the same method without aniline did not show any peak when analysed by fluorescence spectroscopy. To make sure that aniline molecule is indeed inside the shell, we measured the emission spectrum of the supernatant solution obtained after centrifuging the aniline@ $\mathrm{SiO}_{2}$ solution for around six hours at $2000 \mathrm{rpm}$. The nanoshells were precipitated and the supernatant obtained did not show any fluorescence, confirming the fact that the molecules were indeed inside. Note that while preparing the nanobubble, i.e. before the reaction with $\mathrm{CCl}_{4}$ and cyanide, we washed the precipitated silica nanoshells with propanol many times and it is unlikely that there are adsorbed aniline molecules on the silica surface. As said earlier, aniline shows a $\mathrm{pH}$-dependent variation in fluorescence intensity. Hence we monitored the fluorescence intensity by adding micromolar quantities of an acid and a base (figure 6). Aniline is a weak aromatic organic base; the nitrogen has low capacity to hold a proton and hence is a weak base compared to most of the aliphatic amines. The anilinium cation has a $\mathrm{pKa}$ value of 4.63 and at $\mathrm{pH} 2$, aniline is in the form of an anilinium cation which shows less fluorescence intensity compared to that of free aniline. The material shows a change in intensity as the $\mathrm{pH}$ is changed from three to six. However, the change in intensity found in this case was not appreciable

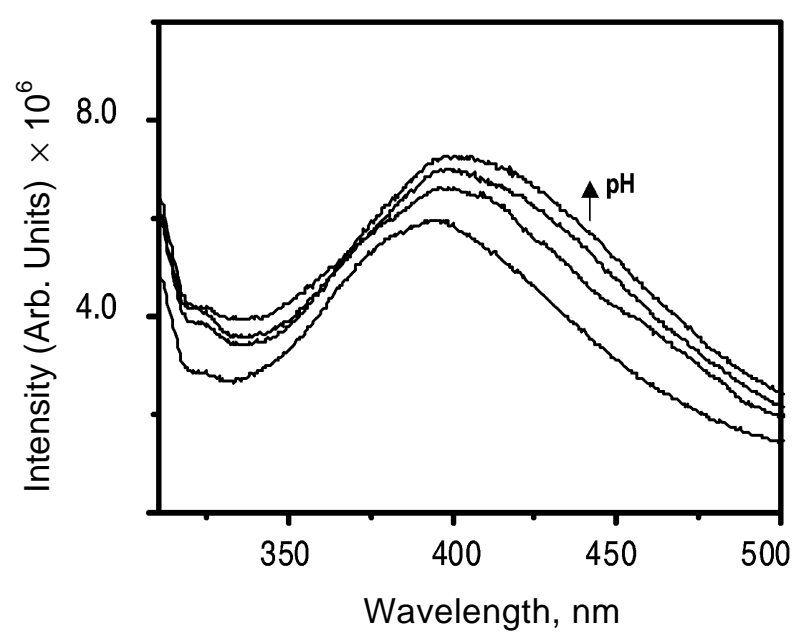

Figure 6. $\mathrm{pH}$ dependent variation in fluorescence intensity of aniline@ $\mathrm{SiO}_{2}$. The time interval given for each measurement is 10 minutes. The excitation wavelength is $290 \mathrm{~nm}$. The $\mathrm{pH}$ was changed from 3 (lowest trace) to 6 (uppermost trace) in these experiments. compared to free aniline even after giving enough time for the penetration of ions (the spectra given in figure 6 were taken after 10 minutes of addition of the base and it showed the same intensity even after $30 \mathrm{~min}$ ). This again points out to the fact that aniline here does not behave in the same way that it does in the free state.

We have characterized this material using cyclic voltammetry. The addition reaction between aniline and cupric chloride solution ${ }^{33}$ was used to study the presence of aniline inside (or outside) the silica shell. Aqueous aniline forms an addition compound when added to cupric chloride solution and the complex shows different electrochemical characteristics with respect to $\mathrm{Cu}$ when compared to free $\mathrm{Cu}^{2+}$ solutions.

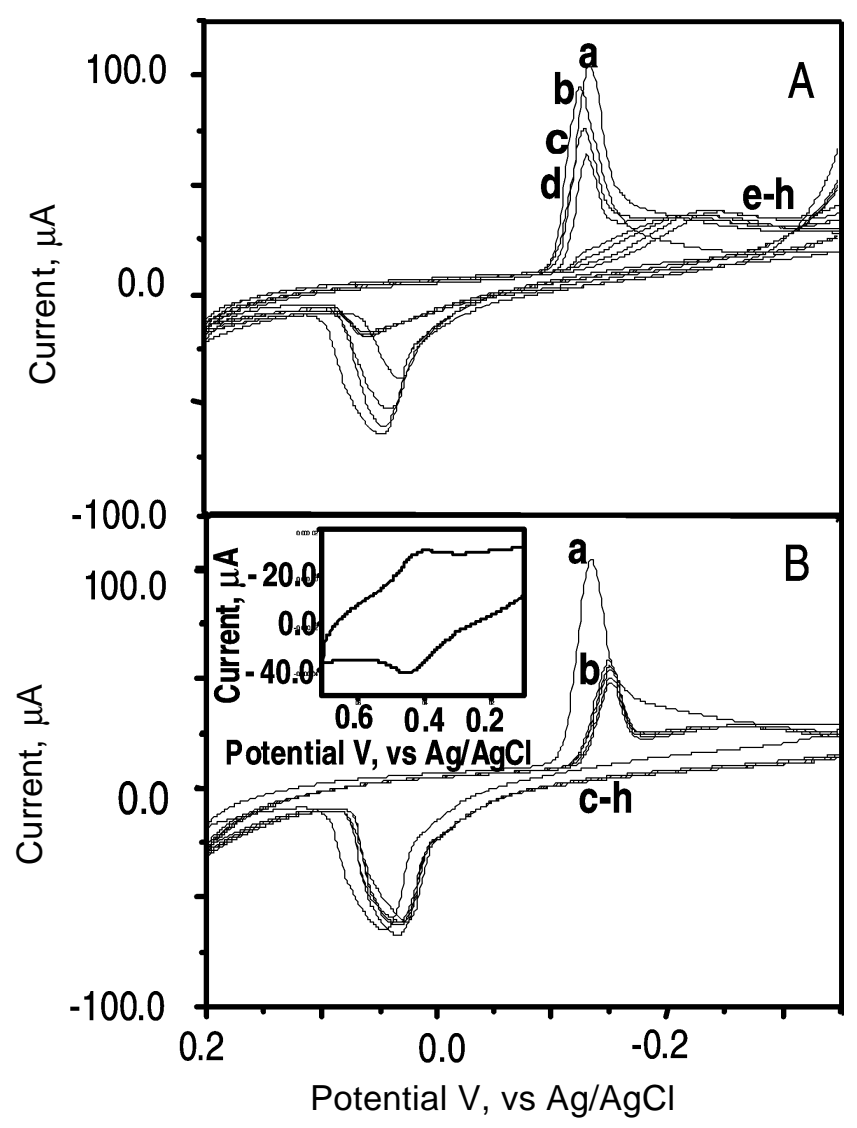

Figure 7. Time-dependent cyclic voltammograms of (A) indicating the addition reaction between aqueous cupric chloride and free aniline solution (concentration $\left.-10^{-4} \mathrm{M}\right)$, and $(\mathrm{B})$ the absence of such reaction between aqueous cupric chloride and aniline@ $\mathrm{SiO}_{2}$ solution taken on $\mathrm{Pt}$ electrode in $0.1 \mathrm{M}$ perchloric acid medium at a sweep rate of $0 \cdot 3 \mathrm{Vs}^{-1}$. Curve a and curves ${ }^{-} \mathrm{b}-\mathrm{h}$ (taken at a time interval of five minutes) show $\mathrm{CV}$ in the absence and presence of aniline respectively. The inset shows the cyclic voltammogram of the aniline@ $\mathrm{SiO}_{2}$ solution at a sweep rate of $0.02 \mathrm{Vs}^{-1}$. 


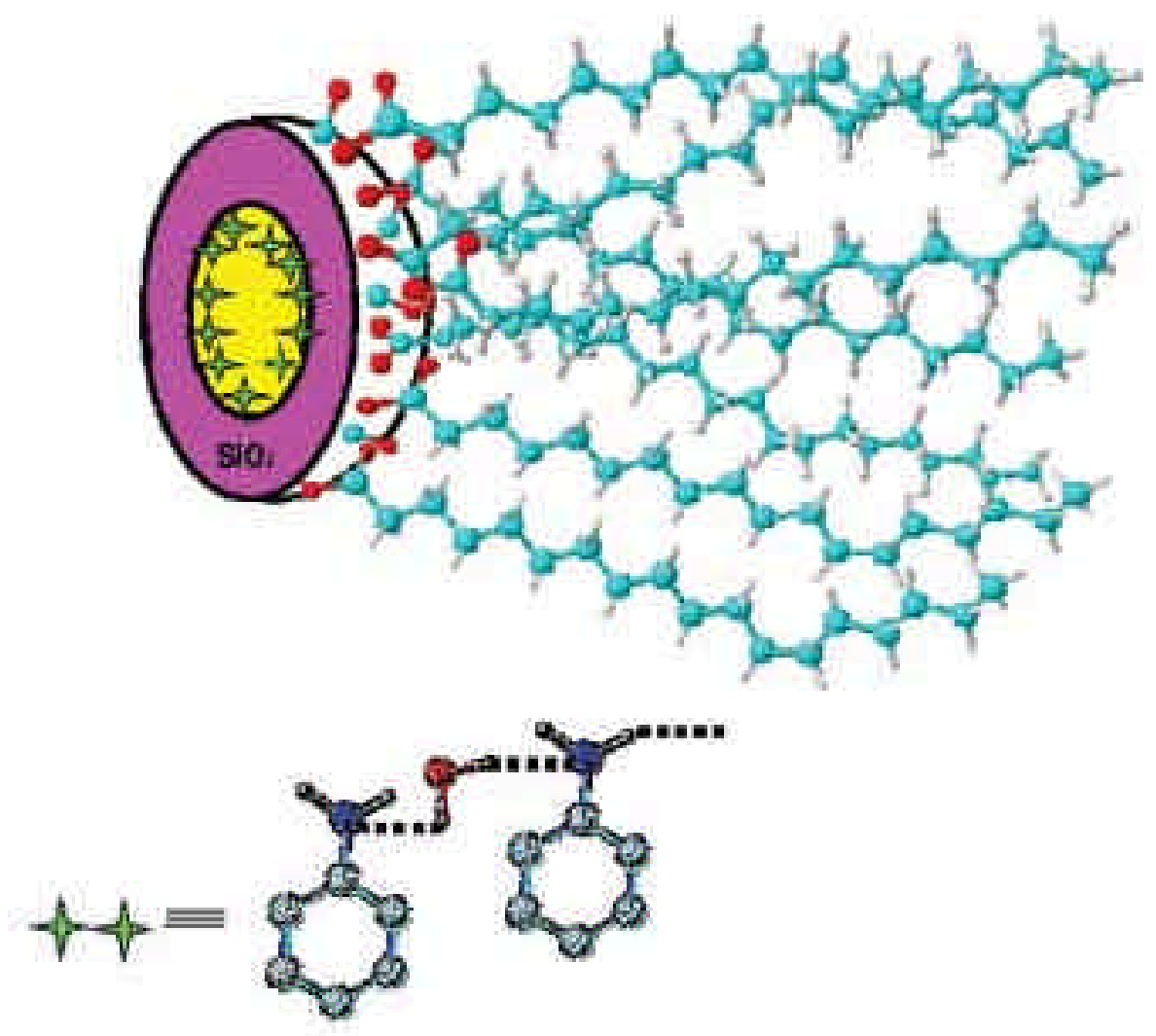

Figure 8. Schematic representation of the cross-section of aniline@ $\mathrm{SiO}_{2} @$ stearate.

This reaction was monitored by cyclic voltammetry on Pt electrode in perchloric acid medium with varying intervals of time for around $35 \mathrm{~min}$. Curve a of figure 7A shows the voltammogram of aqueous cupric chloride solution $\left(10^{-4} \mathrm{M}\right)$ and curves $\mathrm{b}-\mathrm{d}$ show the time-dependent voltammograms obtained subsequently by the addition of aqueous aniline $\left(10^{-4} \mathrm{M}\right)$ to the above solution at a time interval of five minutes. $\mathrm{Cu}$ shows a quasi-reversible redox couple $\mathrm{Cu}^{2+} / \mathrm{Cu}^{0}$ at the cathodic and anodic potentials of -0.135 and $0.046 \mathrm{~V}$, respectively with a peak separation of $0.181 \mathrm{~V}^{34}$ With the addition of aniline solution $\left(10^{-4} \mathrm{M}\right)$, the peak current decreases (curve b-d) and after some time, both the characteristic anodic and cathodic peak potentials shift further. The peak separation also increases to $0.300 \mathrm{~V}$ and with further time interval (curve e-h), the voltammograms become stable indicating the completion of the reaction. The shift of potential and the change in peak separation may be attributed to the distortion of the symmetry of $\mathrm{Cu}^{2+}$ of the molecular species upon addition to aniline. ${ }^{35}$ Different voltammetric behaviour was observed when the same quantity of cupric chloride solution was added to aniline@ $\mathrm{SiO}_{2}$ solution under identical experimental conditions.
Curve a in figure 7B represents cyclic voltammogram of cupric chloride solution $\left(10^{-4} \mathrm{M}\right)$ and curve $\mathrm{b}$ shows the voltammograms after the addition of aniline@ $\mathrm{SiO}_{2}$ solution $\left(10^{-4} \mathrm{M}\right)$ in which an initial decrease in the peak current was noticed (figure 7B, curve b); this phenomenon may be due to an effective deposition of $\mathrm{Cu}$ on the electrode surface catalysed by the presence of other additives in the solution. However, no considerable shift of characteristic anodic and cathodic peak potentials was noted with time (curves $\mathrm{c}-\mathrm{h}$ ) indicating the absence of the reaction found in the case of free aniline. Interestingly, the cyclic voltammogram taken after five days did not show any shift in peak potential, confirming the stability of the system and also showing that the absence of the reaction is not due to the shorter time given for the diffusion of ions through the shell. Silica shell is highly porous in nature hence absence of reaction after five days is rather unexpected. Again it indicates that aniline inside the shell is not in a free form and hence is not available for reaction with cupric chloride. The inset in figure 7B shows cyclic voltammogram of aniline@ $\mathrm{SiO}_{2}$ solution taken at a slow sweep rate of $20 \mathrm{Vs}^{-1}$. The observed $E_{1 / 2}$ value for aniline@ $\mathrm{SiO}_{2}$ is $0.425 \mathrm{~V}$ and for the free ani- 
line $\mathrm{e}^{36,37}$ it is $0.406 \mathrm{~V}$ in the acid medium. It may be noted that the redox accessibility of aniline is achieved through pores of the $\mathrm{SiO}_{2}$ shell; very similar to that of the oxide coated $\mathrm{Au}$ and $\mathrm{Ag}$ nanoparticles. ${ }^{38}$ The study showed that while free aniline is capable of binding to $\mathrm{Cu}^{2+}$, aniline inside the bubble is unavailable for binding.

After confirming the presence of the fluorescing molecule inside the silica shell using absorption and emission spectroscopic studies and voltammetry, we thought of looking at some other parameters such as the solubility of this material in organic solvents. In order to increase its solubility in organic solvents, we functionalized the silica nanoparticle surface using stearic acid. The product obtained after adding millimolar solution of stearic acid to the solution containing aniline@ $\mathrm{SiO}_{2}$ was centrifuged and the precipitate collected was dispersed in 2-propanol. This solution was also found to be fluorescent confirming the fact that aniline is inside the bubble. This material is labeled as aniline@ $\mathrm{SiO}_{2} @$ stearate.

Infrared spectra of the dry material reveals features due to the long alkyl chain and carbonyl group showing that this material is covered with stearate groups (data not shown). ${ }^{39}$ The spectrum shows a peak at around $1650 \mathrm{~cm}^{-1}$ due to carbonyl group present in the stearic acid. ${ }^{40}$ Methylene modes $\left(d_{+}\right.$and $\left.d_{-}\right)$ appear at 2852 and $2925 \mathrm{~cm}^{-1}$ corresponding to disordered polymethylene chains. ${ }^{41}$ The methyl modes $\left(r_{+}\right.$and $r_{-}$) show that there is free rotation possible for the chains. ${ }^{39}$ The spectrum does not show the characteristic progression bands showing that long range order is absent in the chains. The laser desorption mass spectrum (negative mode) of aniline@ $\mathrm{SiO}_{2}$ @ stearate shows a peak at $m / z 283$ due to stearate (data not shown). Keeping all these information we have schematically represented the new material aniline@ $\mathrm{SiO}_{2} @$ stearate as in figure 8. It shows that aniline molecules are inside the shell, surrounded by silica, which in turn is protected with stearate groups, having disordered alkyl chains. The material may contain carbon onion structures if $\mathrm{CCl}_{4}$ was used for the removal of gold core. We have shown earlier that the alkyl chains on core-shell nanomaterials are disordered. $^{20}$

\section{Conclusion}

In this paper we have reported a novel material aniline@ $\mathrm{SiO}_{2}$. It has been characterised thoroughly using UV/VIS absorption spectroscopy, TEM, cyclic voltammetry and emission spectroscopy. Infrared spectroscopic studies show that water is hydrogen bonded within the silica nanoshell. Emission spectrum showed a largely red-shifted band due to the interaction of aniline with the silica nanoshell. This information together with the infrared spectroscopic data suggests that aniline is hydrogen-bonded within the silica nanoshell. Presence of molecules inside the bubble was further confirmed using cyclic voltammetry by monitoring the reaction between cupric chloride and aniline, and it shows that even after giving enough time for the cupper ions to diffuse through the shell there is no reaction occurring with aniline indicating that the molecules are not free for the reaction inside the shell. This material was modified by the functionalization with stearic acid, so that the product is soluble in organic media and can be stored in the dry state. The functionalized material was characterized using infrared spectroscopy and laser desorption ionization mass spectrometry.

\section{Acknowledgements}

TP thanks the Ministry of Information Technology for financial support. The Department of Science and Technology, New Delhi is thanked for equipment support through the Nanoscience and Nanotechnology Initiative. VS thanks the Council of Scientific and Industrial Research, New Delhi for a research associateship. IM would like to thank Prof. Hartmut Fuess for the provision of laboratory facilities.

\section{References}

1. McKiernan J M, Yamanaka S A, Dunn B and Zink J I 1990 J. Phys. Chem. 945652

2. Viteri C R, Gilliland J W and Yip W T 2003 J. Am. Chem. Soc. 1251980

3. Suratwala T, Gardlund Z, Davidson K, Uhlmann D R, Watson J, Bonilla S and Peyghambarian N 1998 Chem. Mater. 10190

4. Van Blaaderen A and Vriji A 1982 Langmuir 82921

5. Verhaegh N A M and Van Blaaderen A 1994 Langmuir 101427

6. Imhof J A, Megens M, Engelberts J J, De Lang D T N, Sprik R and Vos W L 1999 J. Phys. Chem. B103 1408

7. Wheeler J and Thomas J K 1982 J. Phys. Chem. 86 4540

8. Bosma G, Pathmamanoharan C, EHA d H, Kegel K, Van Blaaderen A and Lekkerkerker H N W $2002 J$. Colloid Interface Sci. 245292 
9. Bauer R K, Mayo P D, Ware W R and Wu K C 1982 J. Phys. Chem. 863781

10. Makarova O V, Ostafin A E, Miyoshi H, Jr. Norris J R and Meisel D 1999 J. Phys. Chem. B103 9080

11. Ostafin A E, Siegel M, Wang Q and Mizukami H 2003 Micropor. Mesopor. Mater. 5747

12. Yamanaka S A, Charych D H, Loy D A and Sasaki D Y 1997 Langmuir 135049

13. Flamini A and Panusa A 1997 Sens. Actuators B42 39

14. Sharma A and Wolfbeis O S 1987 Spectrochem. Acta A43 1417

15. Klessinger M and Michl J 1995 Excited states and photochemistry of organic molecules (New York: $\mathrm{VCH}$ )

16. Enustun A and Turkevich B V $1963 \mathrm{~J}$. Am. Chem. Soc. $\mathbf{8 5} 3317$

17. Stöbber W, Fink A and Bohn E 1968 J. Colloid Interface Sci. 2062

18. Nair A S and Pradeep T 2003 Curr. Sci. 841560

19. Rosemary M J, MacLaren I and Pradeep T 2004 Carbon 422352

20. Nair A S, MacLaren I and Pradeep T 2004 J. Mater. Chem. 14857

21. Garvie L A J, Rez P, Alvarez J R and Buseck P R 1998 Sol. Stat. Commun. 106303

22. Calvert J G and Pitts J N Jr 1966 Photochemistry (New York: John Wiley \& Sons)

23. Liz-Marazan L M, Giersig M and Mulvaney P 1996 Langmuir 124329

24. Silverstein R M, Bassler G C and Morrill T C 1963 Spectroscopic investigations of organic compound (New York: John Wiley \& Sons)

25. Neporent B S 1968 Elementary photo processes in molecules (New York: Consultants Bureau)
26. Lakowicz J R 1999 Principles of fluorescence spectroscopy (New York: Kluwer Academic/Plenum)

27. Velic D and Kohler G 2003 Chem. Phys. Lett. 371 483

28. Eimer G A, Gómez Costa M B, Pierella L B and Anunziata O A 2003 J. Colloid Interface Sci. 263 400

29. Hungerford G, Suhling K and Ferreira J A $1999 \mathrm{~J}$. Photochem. Photobiol. A129 71

30. Claracq J, Santos S F C R and Duhamel J 2002 Langmuir 183829

31. Tleugabulova D, Paul J S, Ayers W and Brennan J D 2005 J. Phys. Chem. B109 7850

32. Dennsion S M, Guharay J and Sengupta P K 1999 Spectrochim. Acta A55 1127

33. Labanowska M, Zurowski K R and Bidzinska E 1996 Colloids Surf. A115 297

34. Israel L B, Kariuki N N, Han L, Maye M M, Luo J and Zhong C J 2001 J. Electroanal. Chem. 51769

35. Bacon J and Adams R N 1968 J. Am. Chem. Soc. 90 6596

36. Wawzonek S and Mclntyre T W 1967 J. Electrochem. Soc. 1141025

37. Kim J U, Hartmann W K, Schock H J, Golding B and Nocera D G 1997 Chem. Phys. Lett. 267323

38. Nair A S, Tom R T, Suryanarayanan V and Pradeep T 2003 J. Mater. Chem. 13297

39. Sandhyarani N, Selvam G P, Antony M P and Pradeep T 2000 J. Chem. Phys. 1139794

40. Diyas A M, Sang J A and Kwan K 1996 Bull. Korean Chem. Soc. 17470

41. Sandhyarani N and Pradeep T 2003 Int. Rev. Phys. Chem. 22 221, and references cited therein 